\title{
Influence of Solute Charge and Hydrophobicity on Partitioning and Diffusion in a Genetically Engineered Silk-Elastin-Like Protein Polymer Hydrogel
}

\author{
Adam A. Dinerman, Joseph Cappello, Mohamed El-Sayed, Stephen W. Hoag, \\ Hamidreza Ghandehari*
}

The influence of solute hydrophobicity and charge on partitioning and diffusion in physically crosslinked networks of a genetically engineered SELP polymer was investigated. A series of fluorescent dyes were used to assess the impact of solute charge and hydrophobicity on release behavior. The mechanism of solute release from the SELP hydrogel appeared to vary as a function of dye hydrophobicity. The extent of FITC attachment to amine-terminated G4 dendrimers influenced SELP hydrogel partitioning more than dendrimer diffusion properties. Results suggest the possibility of controlling solute release from SELP hydrogels by modifying the hydrophobicity and surface charge of drugs and drug/polymer conjugates as well as the possibility of "designing-in" solute-specific interactions.

A. A. Dinerman, M. El-Sayed, S. W. Hoag, H. Ghandehari Department of Pharmaceutical Sciences, University of Maryland, $20 \mathrm{~N}$. Pine Street, Baltimore, USA

A. A. Dinerman

Current address: Centocor, Inc., 145 King of Prussia Road, Radnor, PA 19087, USA

M. El-Sayed

Current address: Department of Bioengineering, University of Michigan, 1101 Beal Ave., Ann Arbor, MI 48109, USA

J. Cappello

Protein Polymer Technologies, Inc., 10655 Sorrento Valley Road, San Diego, CA 92121, USA

H. Ghandehari

Departments of Bioengineering and Pharmaceutics and

Pharmaceutical Chemistry, Utah Center for Nanomedicine, Nano Institute of Utah, University of Utah, Salt Lake City, UT 84108, USA

Fax: +1 801585 0575;

E-mail: hamid.ghandehari@pharm.utah.edu

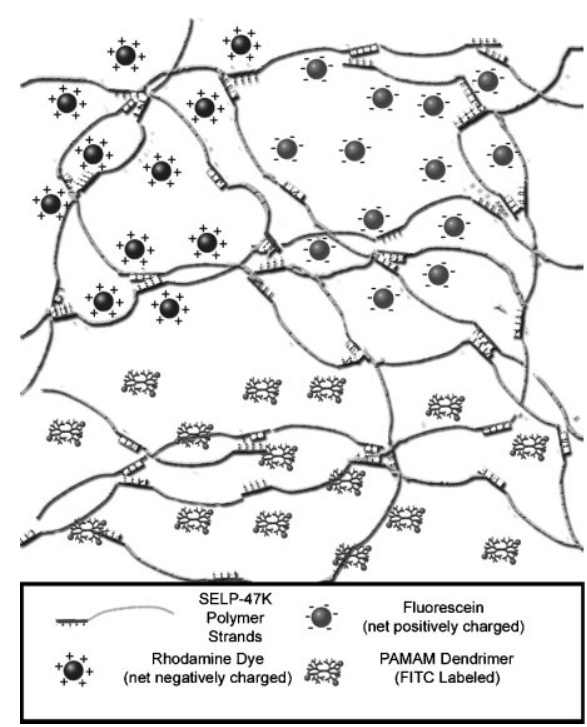

Introduction

Genetically engineered protein polymers represent a class of polymers that can be covalently or physically crosslinked to yield hydrogels. ${ }^{[1-5]}$ Potential advantages of genetically engineered polymers over their synthetic counterparts are tight control of the monomer sequence and narrow molecular weight distribution of the resulting polymer chains. ${ }^{[6]}$ These advantages translate into potential improvements for hydrogels that are intended for use in biomedical applications such as drug delivery where changes in the polymer structure influence the function and fate of the delivery system. Strict control of the monomer sequence provided by genetic engineering may also allow easier manipulation of hydrogel properties such as their degree of equilibrium swelling in the physiological 
environment, the incorporation of stimuli-sensitive or bio-recognizable functional groups, and exact control of polymer crosslinking, all of which influence the rate and the extent of drug release.

Silk-elastin-like protein (SELP)-based polymers are genetically engineered biopolymers composed of repeated sequences of amino acid blocks derived from silk (Gly-AlaGly-Ala-Gly-Ser) and elastin (Gly-Val-Gly-Val-Pro). ${ }^{[7]} \mathrm{Aqu}-$ eous solutions of SELP copolymers are liquid at room temperature allowing bioactive agents to be directly incorporated prior to administration. SELP solutions irreversibly self-assemble under physiological conditions depending on the number of silk-like blocks in the repeat unit. The ability of SELP polymers to form stable, physically crosslinked networks at physiological conditions makes them promising candidates for biomedical applications such as drug delivery. ${ }^{[6,7]}$

Previously we have reported on the influence of polymer concentration, gelation time, and environmental conditions on the equilibrium swelling behavior of a genetically engineered SELP polymer consisting of repeating units of $\left[(\text { GVGVP })_{4} \text { GKGVP }(\text { GVGVP })_{3}(\text { GAGAGS })_{4}\right]_{12}$ named SELP-47K (Table 1). ${ }^{[8]}$ The equilibrium swelling ratio of the physically crosslinked SELP-47K hydrogels did not display temperature sensitivity over the range of $5-50^{\circ} \mathrm{C}$. In addition, the swelling properties were not sensitive to $\mathrm{pH}$ over the range of 2-9 and ionic strength $(0-1 \mathrm{M})$ despite the presence of 12 lysine groups per polymer chain rendering an overall net positive charge on the polymer chains at the test conditions. Essentially, these studies demonstrated that the SELP-47K hydrogel polymer volume fraction does not significantly change as a result of variations in the environmental conditions studied.

In another report, we analyzed the partitioning and diffusion properties of a series of hydrophilic compounds in the size range of most therapeutic small molecules and proteins. ${ }^{[9]}$ We demonstrated that representative hydrophilic compounds in the size range of $180 \mathrm{Da}$ to $13 \mathrm{kDa}$ rapidly diffuse in physically crosslinked networks of SELP$47 \mathrm{~K}$ in a Fickian diffusion-controlled manner. More than a twofold increase in the hydrogel polymer volume fraction (0.04-0.15) in the physically crosslinked system did not appear to dramatically influence the transport rate of a model protein cytochrome $c(13 \mathrm{kDa})$. In addition, it was found that directly loading cytochrome $c$ prior to gelation of the aqueous SELP-47K solution did not appear to significantly alter the release kinetics when compared to data from the equilibrium loaded hydrogels. This result illustrated that the gelation kinetics over a $24 \mathrm{~h}$ period as well as the presence of uncrosslinked SELP-47K (soluble fraction) did not appear to influence the transport behavior of a positively charged protein, namely cytochrome $c$. In another report, a study by Megeed et al. ${ }^{[10]}$ demonstrated that the in vitro release behavior of direct-loaded PRL-CMV DNA from physically crosslinked matrices of SELP-47K is mediated by an ion-exchange mechanism. Release of pRLCMV plasmid DNA at $\mathrm{pH}=7.4$ appeared to be strongly influenced by the ionic strength of the release medium whereby an "on-off" effect was observed when the ionic strength of the medium was changed from 0.15 to $0.01 \mathrm{M}$. These findings appear to suggest that pRL-CMV plasmid DNA interacts via electrostatic interactions with positively charged groups primarily composed of lysine side groups on the polymer backbone.

Often small-molecular-weight drugs are sparingly soluble in physiological media and their charge and hydrophobicity may influence their release behavior from polymeric delivery systems. In addition, protein macromolecules have complex three-dimensional structures possessing exposed surface functional groups. These surface functional groups have the potential to interact with hydrogel polymer chains in a variety of ways (i.e., hydrogen bonding, hydrophobic, and ionic interactions) and could alter the transport behavior in polymeric drug delivery systems. In this study, a series of small molecular weight xanathene dyes (fluorescein, rhodamine B, and rhodamine 6G) were chosen to investigate the influence of solute hydrophobicity on microsolute partitioning and diffusion behavior in SELP-47K hydrogels. We also compared the partitioning and diffusion properties of fluorescein isothiocyanate (FITC)-labeled poly(amidoamine) (PAMAM) dendrimer macrosolutes generation $3(G 3)$ and generation 4 (G4) with an ethylenediamine core. Dendrimers are hyperbranched macromolecules composed of repeated mono-

Table 1. The amino acid sequence (A: Ala, C: Cys, D: Asp, E: Glu, F: Phe, G: Gly, H: His, I: lle, K: Lys, L: Leu, M: Met, N: Asn, P: Pro, Q: Gln, R: Arg, S: Ser, T: Thr, V: Val, W: Trp, Y: Tyr) and molecular weight of the SELP copolymer under study.

\section{Amino acid sequence ${ }^{\text {a) }}$}

Total number of amino acids

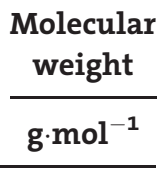

69814

MDVVLQRRDWENPGVTQLNRLAAHPPFASDPMGAGSGAGAGS-

884

$\left[(\text { GVGVP })_{4} \text { GKGVP }(\text { GVGVP })_{3}(\text { GAGAGS })_{4}\right]_{12}(\text { GVGVP })_{4}$ GKGVP $(\text { GVGVP })_{3}-$

(GAGAGS) ${ }_{2}$ GAGAMDPGRYODLRSHHHHHH 
mer groups covalently attached to a core molecule in a radially symmetrical fashion. ${ }^{[11]}$ Since PAMAM dendrimers are water-soluble polymers with a compact, nearly spherical shape, they allow the ability to control probe geometry over a wide range of molecular sizes. For the largest amine terminated dendrimer generation studied (G4), we modified the probe hydrophobicity by varying the number of fluorescent tags attached to the surface of the dendrimers. Finally, we compared the partitioning and diffusion properties of G3 and G4 PAMAM dendrimers with surface hydroxyl groups $(\mathrm{OH})$ to those with an amine functionality at both low and high ionic strength conditions.

\section{Experimental Part}

\section{Materials}

Frozen SELP-47K solutions (or Polymer 47K UBA, 12 wt.-\% were generously provided by Protein Polymer Technologies, Inc. (San Diego, CA). Filtered Milli-O deionized water was used throughout the experiments. Chlorotrimethylsilane and dichloromethane were purchased from Aldrich (Milwaukee, WI) and used to prepare the silanating solution. Triethylamine (TEA) and N,N-dimethylformamide (DMF) was purchased from EM Science (Gibbstown, NJ). The fluorescein derivatives, sodium fluorescein (MW 367), rhodamine B (MW 473), and rhodamine 6G (MW 479) were purchased from Sigma Chemical Co. (St. Louis, MO). Hydroxyl- and amineterminated PAMAM dendrimers and FITC isomer I were purchased from Sigma Chemical Co.. All other chemicals were reagent grade and purchased from Sigma Chemical Co.

\section{Fluorescence Labeling and Purification of PAMAM Dendrimers}

Amine terminated dendrimers were fluorescent-labeled following the procedure described previously. ${ }^{[12]}$ Briefly, a methanolic solution (as supplied) of an amine-terminated dendrimer was diluted in a $1 \times$ PBS solution (approx. 1:100 dilution). An appropriate amount of FITC was dissolved in acetone based on the desired initial PAMAM dendrimer/FITC molar ratio. The acetone solution containing the FITC was then added dropwise into a PBS solution $(\mathrm{pH}=7.4)$ containing the dendrimers under stirring conditions. The labeling reaction was allowed to continue for $24 \mathrm{~h}$ at room temperature.

Hydroxyl-terminated dendrimers were fluorescent tagged by reaction of surface hydroxyl groups with FITC. In order to make the hydroxyl groups more reactive, labeling was performed in a polar aprotic solvent DMF and TEA was used as a catalyst. Prior to using the unlabeled hydroxylated dendrimers, methanol was removed from these dendrimer solutions by solvent evaporation at $70{ }^{\circ} \mathrm{C}$. Hydroxyl-terminated dendrimers were then re-dissolved in water and lyophilized. Lyophilized hydroxyl-terminated dendrimers were dissolved in DMF (approx. 1:10 w/v dilution). FITC and TEA were added to the reaction mixture using an initial molar ratio of 1:1:1 (dendrimer/FITC/TEA). FITC was dissolved in DMF and added drop-wise into the reaction mixture under stirring. TEA was also added drop-wise into the reaction mixture. The reaction continued for four days at $95-100{ }^{\circ} \mathrm{C}$ under nitrogen.

Following the labeling reactions, all dendrimer reaction mixtures were extensively dialyzed against deionized water. Dialyzed solutions were then fractionated using a Superose 12 HR 16/50 preparative size exclusion column on a fast protein liquid chromatography (FPLC) system (Amersham Bioscience, Piscataway, NJ). A mobile phase consisting of 30:70 vol.-\% acetonitrile and Tris buffer $(\mathrm{pH}=8)$ was used at a flow rate of $1 \mathrm{~mL} \cdot \mathrm{min}^{-1}$ with $\mathrm{UV}$ detection at $280 \mathrm{~nm}$. At designated times fractions corresponding to dendrimers were collected and pooled for further dialysis against deionized water. The fractionated dendrimers were then lyophilized and stored at $-20^{\circ} \mathrm{C}$ prior to use. The extent of FITC labeling on PAMAM dendrimers is expressed as mmol FITC per gram of polymer and was determined spectrophotometrically at $495 \mathrm{~nm}$ using free FITC as a standard.

\section{Preparation of Hydrogels}

SELP-47K hydrogels were prepared as previously described. ${ }^{[8]}$ Briefly, frozen 12 wt.-\% SELP-47K solutions were thawed by immersing polymer-containing syringes into a room-temperature water bath for $5 \mathrm{~min}$. Thawed solutions were slowly injected into gel molds consisting of a silicone gasket (ca. $1.6 \mathrm{~mm}$ thickness) sandwiched between two silanated glass slides secured by binder clips. Filled gel molds were placed into a temperature controlled convection incubator (Precision, Winchester, VA) at $37^{\circ} \mathrm{C}$ for $24 \mathrm{~h}$. Following incubation, gel disks (thickness $\approx 1.6 \mathrm{~mm}$, diameter $\approx 6.0 \mathrm{~mm}$ ) were obtained using a cork borer. The soluble polymer fractions remaining in the hydrogels post-gelation were removed prior to solute loading and diffusion studies. Hydrogel disks were washed for 1 week in $1 \times \mathrm{PBS}\left(10^{-2} \mathrm{M} \mathrm{PBS}, \mathrm{pH}=7.4\right.$, $\mu=0.15)$ with $0.01 \% \mathrm{w} / \mathrm{v}$ sodium azide under mild agitation (120 rpm) in a temperature controlled convection incubator (VWR, Model 1575, Bridgeport, NJ) set at $37^{\circ} \mathrm{C}$. Fresh buffer was replaced daily throughout the washing period. Prior to use, washed hydrogel disks were stored at 2 to $8^{\circ} \mathrm{C}$ in $1 \times$ PBS with $0.01 \% \mathrm{w} / \mathrm{v}$ sodium azide.

\section{Diffusion Studies}

Washed hydrogel disks were initially placed into concentrated solutions of fluorescent-labeled dendrimers ( 0.1 and $\left.1 \times 10^{-3} \mathrm{M}\right)$ and fluorescein derivatives $\left(10^{-4} \mathrm{M}\right)$, prepared in phosphatebuffered solutions $(\mathrm{pH}=7.4)$ with $0.01 \% \mathrm{w} / \mathrm{v}$ sodium azide at $37^{\circ} \mathrm{C}$ for $48 \mathrm{~h}$. Samples of the loading solutions were taken prior to and after solute loading for concentration determination. No visible precipitation of the xanathene dyes or fluorescent labeled dendrimers was observed in the loading solutions following the equilibration period. Size-exclusion chromatography was also used to confirm the stability of fluorescent-labeled dendrimers post-loading.

For the release studies, loaded hydrogel disks were placed in a $4 \mathrm{~mL}$ vial containing $3 \mathrm{~mL}$ of a phosphate-buffered solution with $0.01 \% \mathrm{w} / \mathrm{v}$ sodium azide $(\mathrm{pH}=7.4)$ and placed in a temperature 
controlled shaker bath set at $37^{\circ} \mathrm{C}$. At designated time points, samples were removed and replaced with fresh buffer to maintain sink conditions. Rhodamine B and rhodamine $6 \mathrm{G}$ were analyzed using a UV/Vis spectrophotometer (Pharmacia Ultrospec 240) at $\lambda=553$ and $526 \mathrm{~nm}$, respectively. Fluorescent-labeled dendrimers and fluorescein were analyzed using a fluorescence detection method at $\lambda_{\mathrm{ex}}=485 \mathrm{~nm} / \lambda_{\mathrm{em}}=535 \mathrm{~nm}$ utilizing a multilabel plate reader (Wallac Victor ${ }^{2}$, Boston, MA).

Following the release studies, hydrogel disk dimensions were obtained using calipers (Brown \& Sharpe, Kingston, RI). Mass balances were performed on all depleted hydrogel disks to determine the amount of solute irreversibly bound (unable to be released) to the SELP hydrogels. To perform a mass balance, depleted hydrogel disks were dissolved in $0.2 \mathrm{~mL}$ of 88 vol.-\% formic acid and the $\mathrm{pH}$ of the dissolved gel solution was adjusted to 7.4 with $1 \mathrm{~N} \mathrm{NaOH}$. The volume of mass balance solutions was adjusted to $5 \mathrm{~mL}$ using phosphate buffer with $0.01 \% \mathrm{w} / \mathrm{v}$ sodium azide $(\mathrm{pH}=7.4)$ and analyzed at the corresponding wavelengths for the model compounds (see above). Recovery rates were determined by spiking dissolved gel solutions with known amounts of solute.

Solute partition coefficients were determined from the equilibrium loading studies using Equation (1), where $M_{\infty}$ is the total amount of solute released at infinite time, $m_{\text {gel }}$ is the swollen hydrogel weight, $\rho_{\text {gel }}$ is the density of the hydrogel $\left(\rho_{\text {gel }}=1.013 \mathrm{~g} \cdot \mathrm{cm}^{-3}\right)$ and $C_{\text {soln }}$ is the final concentration of the solution after loading:

$$
K=\frac{C_{\text {gel }}}{C_{\text {soln }}}=\frac{M_{\infty}}{\left(m_{\text {gel }} / \rho_{\text {gel }}\right) C_{\text {soln }}}
$$

Effective diffusivities $\left(D_{\mathrm{e}}\right)$ were determined by nonlinear regression of the experimental release curves $\left(M_{t} / M_{\infty}\right.$ vs. $\left.t\right)$ to the mathematical solution for two-dimensional Fickian diffusion in a tablet geometry using Mathematica ${ }^{\mathbb{R}}$ version 4.0. ${ }^{[13,14]}$ The mathematical model shown in Equation (2) assumes an initial, uniform distribution of solute in the hydrogel disks, diffusional transport in the radial $(r)$ and axial $(z)$ directions only, a concentration-independent effective diffusivity, negligible mass transfer resistances at the gel surface, and sink conditions in the release medium. In Equation (2), $M_{t}$ represents the cumulative amount of solute released at time $t, M_{\infty}$ refers to the cumulative amount of solute released at infinite time, $r$ is the radius of the hydrogel disk, $l$ is the thickness of the hydrogel disk, $\alpha_{i}$ are the roots of the Bessel function of the first kind of order zero, $J_{0}\left(\alpha_{i}\right)=0$,

$$
\begin{aligned}
\frac{M_{t}}{M_{\infty}}= & 1-\frac{32}{\pi^{2}} \sum_{n=1}^{\infty} \frac{1}{\alpha_{n}^{2}} \exp \left(-\frac{\alpha_{n}^{2}}{r^{2}} D_{\mathrm{e}} t\right) \\
& \times \sum_{p=0}^{\infty} \frac{1}{(2 p+1)^{2}} \exp \left(-\frac{(2 p+1)^{2} \pi^{2}}{l^{2}} D_{\mathrm{e}} t\right)
\end{aligned}
$$

\section{Equilibrium Binding Studies}

Pre-weighed hydrogels were placed into a series of concentrated FITC-labeled dendrimer solutions and allowed to equilibrate for $48 \mathrm{~h}$ at $37^{\circ} \mathrm{C}$. The amount of mobile dendrimer loaded into each hydrogel was determined by desorption from loaded hydrogels into a PBS solution $(\mathrm{pH}=7.4,[\mathrm{NaCl}]=0.500 \mathrm{M})$. The total amount of dendrimer loaded (free + bound) per volume of swollen hydrogel $\left(C_{\text {gel }}\right)$ was determined at each loading concentration. The amount of dendrimer irreversibly bound to the hydrogel matrix was assessed via a mass balance on the gel disks following desorption. All dendrimer binding studies were performed in triplicate at each respective loading concentration. The total amount of PAMAM dendrimer bound to the polymer network $\left(c_{b}\right)$ was determined using Equation (3) by accounting for the presence of free dendrimer in the aqueous portion of the network, where $\varepsilon$ represents the estimated volume fraction of bulk water in the hydrogel,

$$
c_{\mathrm{b}}=C_{\mathrm{gel}}-\varepsilon_{\varepsilon}
$$

\section{Octanol/Buffer Partition Coefficients $\left(\mathrm{K}_{\mathrm{ob}}\right)$}

Octanol/buffer partition coefficients were determined by equilibrating overnight a known concentration of solute in PBS ( $\mathrm{pH}=7.4$, $\mu=0.15$ ) with an equal volume of octanol. The octanol was preequilibrated with PBS prior to experiments. The amount of solute present in the octanol phase was determined by performing a material balance on the solute remaining in the aqueous phase (buffer) following the equilibration period.

\section{Statistical Analysis}

Single factor analysis of variance (ANOVA) at $\alpha=0.05$ was used to determine statistically significant differences between test samples. All studies were performed in triplicate unless otherwise noted.

\section{Results and Discussion}

\section{Influence of Fluorescent Dye Structure on Partitioning, Diffusion, and Release Behavior}

Structurally similar fluorescent dyes fluorescein, rhodamine $B$, and rhodamine $6 \mathrm{G}$ were used to explore the partitioning and diffusion of small molecules of similar size in Polymer 47K hydrogels. As shown in Table 2, the fluorescent dyes represent varying net charges at physiological $\mathrm{pH}$ and relative hydrophobicities as demonstrated by their octanol/buffer partition coefficients. Release profiles for the fluorescent dyes are presented in Figure 1a. Despite their similar molecular size, the compounds represented distinctly different release behavior in physiological buffer with an initial release rate order of fluorescein $>$ rhodamine B $>$ rhodamine 6G. As shown in Table 3, the estimated effective diffusivity (assuming only diffusional mass transport) calculated from the fraction released versus time profile sharply decreases as a result of functional group modifications in the fluorescein structural unit. The results in Table 3 indicate that not only changes in diffusivity occur as the functional groups are modified on 
Table 2. Molecular size and physicochemical properties of small molecules with basic structure.<smiles></smiles>

‥

\begin{tabular}{|c|c|c|c|c|}
\hline \multirow[t]{2}{*}{ Compound } & \multirow[t]{2}{*}{ Substituents } & $\begin{array}{c}\text { Molecular } \\
\text { weight }\end{array}$ & \multirow[t]{2}{*}{$\begin{array}{l}\text { Net charge } \\
\text { at } \mathrm{pH}=7.4\end{array}$} & \multirow[t]{2}{*}{$K_{\text {octanol/buffer }}$} \\
\hline & & $\mathrm{g} \cdot \mathrm{mol}^{-1}$ & & \\
\hline fluorescein & $R_{1}=\mathrm{O}, R_{2}=\mathrm{H}, R_{3}=\mathrm{COO}^{-}$ & 376 & - & $0.6^{\mathrm{a})}$ \\
\hline rhodamine B & $R_{1}=\mathrm{N}\left(\mathrm{C}_{2} \mathrm{H}_{5}\right)_{2}, R_{2}=\mathrm{H}, R_{3}=\mathrm{COO}^{-}$ & 479 & amphoteric & $274^{\mathrm{a})}$ \\
\hline rhodamine $6 G$ & $R_{1}=\mathrm{NHC}_{2} \mathrm{H}_{5}, R_{2}=\mathrm{CH}_{3}, R_{3}=\mathrm{COOC}_{2} \mathrm{H}_{5}$ & 451 & + & 299 \\
\hline
\end{tabular}

a) Octanol/buffer partition coefficients taken from ref. ${ }^{[22]}$

the fluorescein structural unit but the compounds appear to interact more with the polymer chains in the hydrogel network as evident by a large increase in the gel-solute equilibrium partition coefficient. An increase in hydrogel partitioning appears to correlate with the more hydrophobic dyes (high $K_{\mathrm{ob}}$ ) and may suggest the presence of hydrophobic interactions with the hydrophobic amino acid residues of the polymer chains. The release behavior could also be visually observed due to the color associated with the fluorescent dyes. Fluorescein appeared to demonstrate a Fickian diffusion process whereby a diffusion front moved from the surface of the hydrogel inwards over the course of the release experiments. In contrast, the release behavior of the more hydrophobic dyes (rhodamine B and rhodamine $6 \mathrm{G}$ ) did not demonstrate a visually distinct diffusion front. Hydrogels loaded with the rhodamine dyes initially displayed a uniform, brightly colored appearance that uniformly decreased in intensity throughout the release experiments,

$$
\frac{M_{t}}{M_{\infty}}=k t^{n}
$$

Using the method of Ritger and Peppas the release index, $n$ was calculated based on Equation (4) to characterize the overall dye release behavior from the hydrogel matrix.$^{[15]}$ In general, when $n$ is 0.5 the dominant release mechanism is Fickian diffusion, anomalous diffusion is dominant when $0.5<n<1.0$, case II transport (zero order) is observed when $n=1.0$, and when $n>1.0$ super-case II transport is the dominant release mechanism. As a result of their partitioning ability, the dyes appear to represent signifi- cantly different release mechanisms. Fluorescein appears to exhibit more Fickian diffusion behavior, while rhodamine $B$ exhibits anomalous diffusion, and rhodamine 6G represents super case II transport. Since dye release behavior appears to be strongly influenced by its interaction with SELP-47K (dominant mode of release is not Fickian diffusion), the tabulated estimated effective diffusivities based on a Fickian release mechanism should only be utilized for comparison and not regarded as "true" effective diffusivities.

The microsolute fluorescein has a net negative charge at physiological $\mathrm{pH}$ and displays favorable partitioning behavior with the polymer chains in the hydrogel. Despite the significant interactions with polymer chains, fluorescein release behavior displays essentially Fickian release characteristics at physiological conditions (Figure 1b). As a result, we investigated the influence of electrostatic interactions with the positively charged groups in the polymer backbone and its effect on the effective solute diffusivity in hydrogels. A decrease in the ionic strength of the release buffer from 0.500 to $0 \mathrm{M} \mathrm{NaCl}$ lowered the effective diffusivity of fluorescein (Table 3). It should be noted that these experiments were performed under equimolar loading conditions $\left(10^{-4} \mathrm{M}\right.$ fluorescein) and a tenfold decrease in loading solution concentration $\left(10^{-5} \mathrm{M}\right)$ did not significantly alter the release or solute partitioning behavior (data not shown). Essentially, this information indicates that the extent of fluorescein bound to the polymer chains at $10^{-4} \mathrm{M}$ loading does not appreciably change with a decrease in the amount of fluorescein present in the bulk solution. It is apparent from Figure $1 \mathrm{~b}$ that the so-called "burst-effect" for fluorescein is reduced in low ionic strength buffer. The burst effect is most likely related 

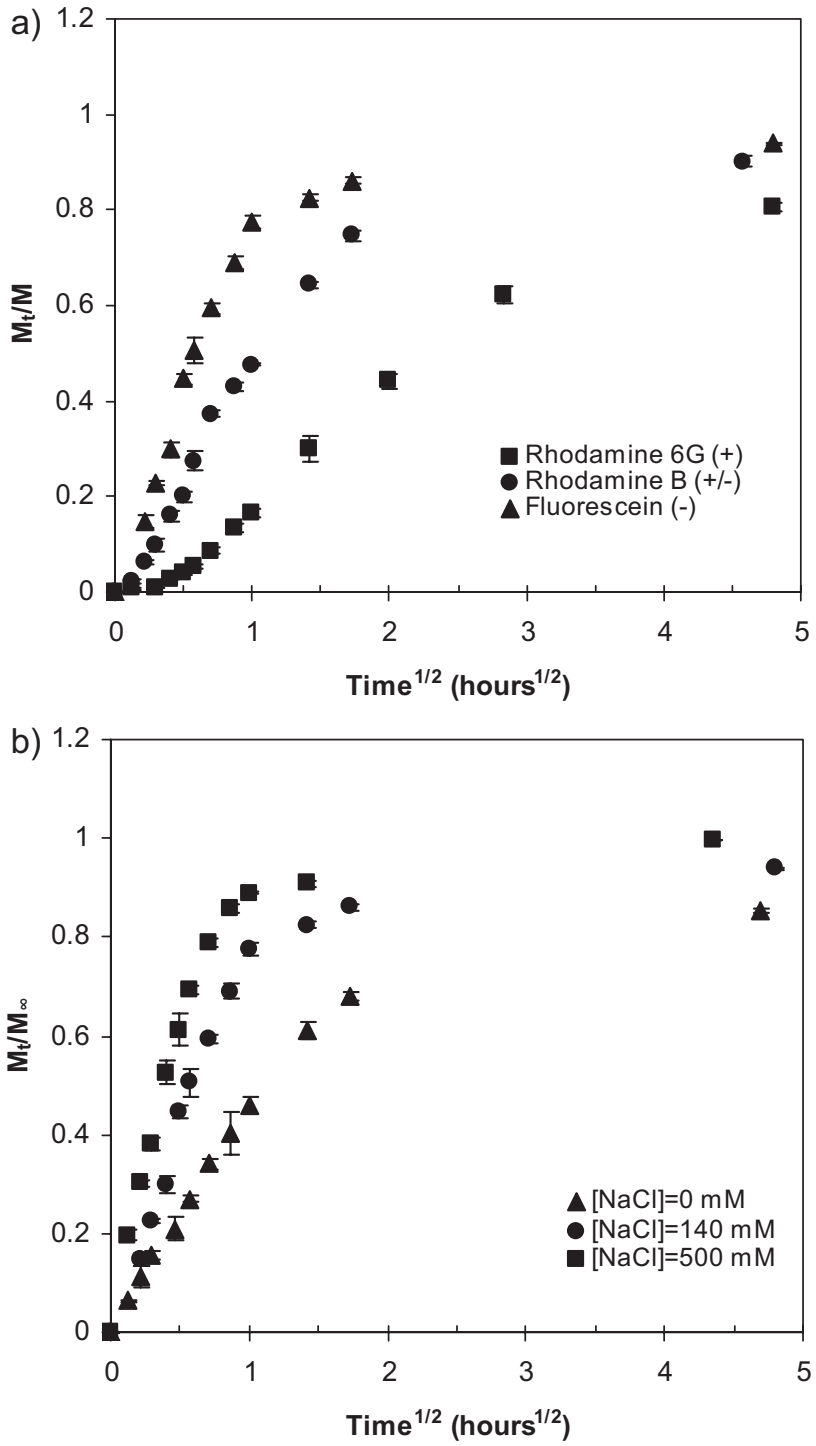

Figure 1. Fraction released versus square root of time for fluorescent dyes released from SELP-47K hydrogels in PBS $(\mathrm{pH}=7.4, \mu=0.15)$. (a) Influence of dye structure on release behavior. (b) Influence of ionic strength on fluorescein release behavior. All hydrogels equilibrium loaded from $10^{-4} \mathrm{M}$ solute loading solutions. Symbols represent mean value \pm 1 standard deviation $(n=3)$.

to the initial release of free fluorescein in the aqueous pores, which is then followed by the release of fluorescein electrostatically interacting with positively charged groups on the polymer backbone. As the ionic strength of the release medium increases, fluorescein is more readily displaced from the hydrogel system as a result of competition from counterions in solution with cationic groups on the polymer backbone. Since fluorescein was loaded at an ionic strength of $0.15 \mathrm{M}$, the extent of fluorescein initially bound would appear to be low. Although the release rate is appreciably reduced in low ionic strength buffer, the calculated release index indicates a dominant Fickian diffusion mechanism (Table 3). These experimental results suggest that fluorescein most likely interacts with polymer chains (via electrostatic interactions) presumably located in the vicinity of the aqueous pores of the crosslinked Polymer 47K network. Although the observed interaction appears to be relatively weak, these results suggest that the release behavior of other negatively charged, small molecular weight, compounds can potentially be controlled by taking advantage of their electrostatic interactions with the SELP hydrogel.

\section{Influence of Amine-Terminated PAMAM Dendrimers and the Extent of Labeling on Partitioning and Diffusion in SELP-47K Hydrogels}

The physicochemical properties of the FITC labeled PAMAM dendrimers may be found in Table 4. The partitioning and diffusion results for $\mathrm{G} 3-\mathrm{NH}_{2}$ (1:1) and G4- $\mathrm{NH}_{2}$ (1:1) at physiological conditions is presented in Table 5. Representative release profiles are presented in Figure $2 \mathrm{a}$ and indicate a Fickian diffusion controlled process similar to that previously observed for transport in this SELP hydrogel. ${ }^{[9]}$ In addition, the estimated effective diffusivities correlated well with those of other hydrophilic solutes determined previously in $12 \mathrm{wt} . \%$ SELP-47K cured for $24 \mathrm{~h}$ at $37^{\circ} \mathrm{C}$. It is worth noting that the extent of FITC labeled on both probes differed due to the size of each dendrimer. However, the apparent difference in labeling efficiency did not correspond to significant changes in $K_{\mathrm{ob}}$ or the partitioning behavior of either probe in the hydrogel.

Figure $2 \mathrm{~b}$ and Table 5 present the partitioning and diffusion data for FITC labeled $\mathrm{G} 4-\mathrm{NH}_{2}$ with increasing amounts of FITC substituted surface groups. The effect of doubling the initial FITC labeling of $\mathrm{G} 4-\mathrm{NH}_{2}$ from 1:1 to 2:1 corresponded to approximately a twofold increase in $K_{\mathrm{ob}}$. Further increases in the extent of labeling did not appreciably change $K_{\mathrm{ob}}$ (relative hydrophobicity) and may be the result of the overwhelming contribution of the PAMAM dendrimer to its physicochemical properties. Despite increases in the extent of surface groups labeled with FITC, diffusion behavior in the hydrogels remained essentially unchanged. In contrast to the diffusion results for $10^{-4} \mathrm{M}$ loading conditions, G4-NH2 dendrimer hydrogel partitioning increased with approximately a fourfold increase in the extent of labeling for $\mathrm{G} 4-\mathrm{NH}_{2}$ from 1:1 to 4:1 initial molar ratio. Also note that the apparent diffusivity is almost half of that observed for $\mathrm{G} 4-\mathrm{NH}_{2}$ (1:1) at $10^{-4}{ }_{M}$ loading. Since the entire release curve was used to estimate $D_{\mathrm{e}}$, we believe this difference is most likely due to a combination of low initial loading and the presence of more FITC groups at the surface of G4- $\mathrm{NH}_{2}(2: 1)$, G4- $\mathrm{NH}_{2}(4: 1)$, and $\mathrm{G} 4-\mathrm{NH}_{2}$ (8:1) dendrimers. As a result, 
Table 3. Influence of release medium ionic strength on the equilibrium partition coefficient $(K)$, effective diffusivity $\left(D_{\mathrm{e}}\right)$, and release index $(n)$ for fluorescein $(-)$ in $12 \mathrm{wt}$. $\%$ Polymer $47 \mathrm{~K}$ hydrogels. All hydrogels were prepared from $12 \mathrm{wt} .-\% \mathrm{SELP}-47 \mathrm{~K}$ solutions and equilibrated in $10^{-4} \mathrm{M}$ loading solution using PBS $(\mathrm{pH}=7.4, \mu=0.15)$.

\begin{tabular}{|c|c|c|c|c|c|}
\hline $\begin{array}{l}\text { Release buffer } \\
{[\mathrm{NaCl}]}\end{array}$ & Solute & $K$ & $D_{\mathrm{e}}$ & $K D_{\mathrm{e}}$ & $n$ \\
\hline $10^{-3} M$ & & & $10^{-7} \mathrm{~cm}^{2} \cdot \mathrm{s}^{-1}$ & $10^{-7} \mathrm{~cm}^{2} \cdot \mathrm{s}^{-1}$ & \\
\hline 0 & fluorescein & $3.12 \pm 0.16$ & $1.49 \pm 0.14$ & $4.61 \pm 0.24$ & $0.47 \pm 0.01$ \\
\hline 140 & fluorescein & $2.9 \pm 0.11$ & $5.02 \pm 0.13$ & $14.54 \pm 0.58$ & $0.58 \pm 0.04$ \\
\hline 140 & rhodamine B & $19.59 \pm 1.71$ & $1.63 \pm 0.08$ & $32.06 \pm 4.04$ & $0.75 \pm 0.01$ \\
\hline 140 & rhodamine $6 \mathrm{G}$ & $31.11 \pm 1.68$ & $0.29 \pm 0.02$ & $8.95 \pm 1.02$ & $1.91 \pm 0.01$ \\
\hline 500 & fluorescein & $2.98 \pm 0.23$ & $12.25 \pm 0.54$ & $36.36 \pm 2.79$ & $0.46 \pm 0.04$ \\
\hline
\end{tabular}

the cumulative amount released from hydrogels equilibrated in $10^{-4} \mathrm{M}$ loading solutions resulted in a greater influence on the amount released at long release times. The increased partitioning at low concentrations as a function of the extent of FITC labeling indicates that the FITC substituted functional groups contribute to the partitioning behavior. These results appear to suggest that the attachment of hydrophobic drugs to these dendrimers (polymer/drug conjugates) will influence partitioning in the SELP hydrogels.

A tenfold increase in the concentration of the loading solution $\left(10^{-3} \mathrm{M}\right)$ resulted in reduced hydrogel partitioning for all of the $\mathrm{G} 4-\mathrm{NH}_{2}$ dendrimers regardless of the extent of FITC labeling. As a result, it appears that dendrimer/dendrimer repulsion may be responsible for the decreased partitioning ability at higher loading concentrations. This is supported by the fact that the partition coefficients changed without a significant change in the effective diffusivity over the series of G4-
$\mathrm{NH}_{2}$ dendrimers at $10^{-4} \mathrm{M}$ loading conditions. If reduced partitioning was more related to changes in dendrimer size, a decrease in the effective diffusivity would be expected if the dendrimers formed dimers or higher order soluble aggregates.

\section{Influence of PAMAM Dendrimer Surface Charge and Surface Group Functionality on Partitioning and Diffusion in SELP-47K Hydrogels}

Results for the $\mathrm{G} 4-\mathrm{NH}_{2}$ dendrimers with an increased number of surface FITC groups suggest that extent of hydrophobic drug attachment plays a dominant role in PAMAM dendrimer solute partitioning behavior with SELP47K hydrogels at low loading conditions. To further explore this phenomenon, we covalently attached FITC to G3 and G4 dendrimers with a hydroxyl surface functionality (G3$\mathrm{OH}$ and $\mathrm{G} 4-\mathrm{OH})$. As shown in Table 4, the unlabeled

Table 4. Molecular size and physicochemical properties of PAMAM dendrimer macrosolutes.

\begin{tabular}{|c|c|c|c|c|c|}
\hline \multirow[t]{2}{*}{$\begin{array}{l}\text { Compound } \\
\text { (initial FITC/polymer) }\end{array}$} & $\begin{array}{c}\text { Theoretical } \\
\text { molecular weight }\end{array}$ & $R_{\text {theory }}{ }^{\text {a) }}$ & $\begin{array}{l}\text { Extent of } \\
\text { labeling }\end{array}$ & $K_{\mathrm{ob}}$ & $\begin{array}{l}\text { Aqueous } \\
\text { diffusivity, } D_{0}\end{array}$ \\
\hline & $\mathrm{g} \cdot \mathrm{mol}^{-1}$ & $\AA$ & $\begin{array}{c}\text { mmol } \\
\text { FITC } \cdot(\mathrm{g} \text { polymer })^{-1}\end{array}$ & & $10^{6} \mathrm{~cm}^{2} \cdot \mathrm{s}^{-1 \mathrm{~b})}$ \\
\hline G3- $\mathrm{NH}_{2}(1: 1)$ & 6909 & 18.0 & 0.10 & 0.2 & 1.83 \\
\hline G4- $\mathrm{NH}_{2}(1: 1)$ & 14215 & 22.7 & 0.03 & 0.2 & 1.45 \\
\hline G4- $\mathrm{NH}_{2}(2: 1)$ & 14215 & 22.7 & 0.11 & 0.5 & 1.45 \\
\hline G4- $\mathrm{NH}_{2}(4: 1)$ & 14215 & 22.7 & 0.20 & 0.5 & 1.45 \\
\hline $\mathrm{G} 4-\mathrm{NH}_{2}(8: 1)$ & 14215 & 22.7 & 0.44 & 0.4 & 1.45 \\
\hline G3-OH (1:1) & 6941 & 18.0 & 0.10 & 0.1 & 1.83 \\
\hline G4-OH (1:1) & 14279 & 22.7 & 0.01 & 0.2 & 1.45 \\
\hline
\end{tabular}

a) Solute radius was calculated from estimated aqueous diffusivities using the Stokes-Einstein equation; ${ }^{\text {b) }}$ Aqueous diffusivities estimated from theoretical molecular weight of unlabeled dendrimers using $D=260 \bar{M}_{\mathrm{w}}^{-1 / 3}$ found in ref. ${ }^{[23]}$ and corrected for $37^{\circ} \mathrm{C}$. 
Table 5. Influence of PAMAM dendrimer properties on the equilibrium partition coefficient $(K)$, effective diffusivity $\left(D_{\mathrm{e}}\right)$, and permeability $\left(K D_{\mathrm{e}}\right)$ in $12 \mathrm{wt}$. $\%$ SELP-47K hydrogels. All hydrogels were prepared from $12 \mathrm{wt}$. \% SELP-47K solutions.

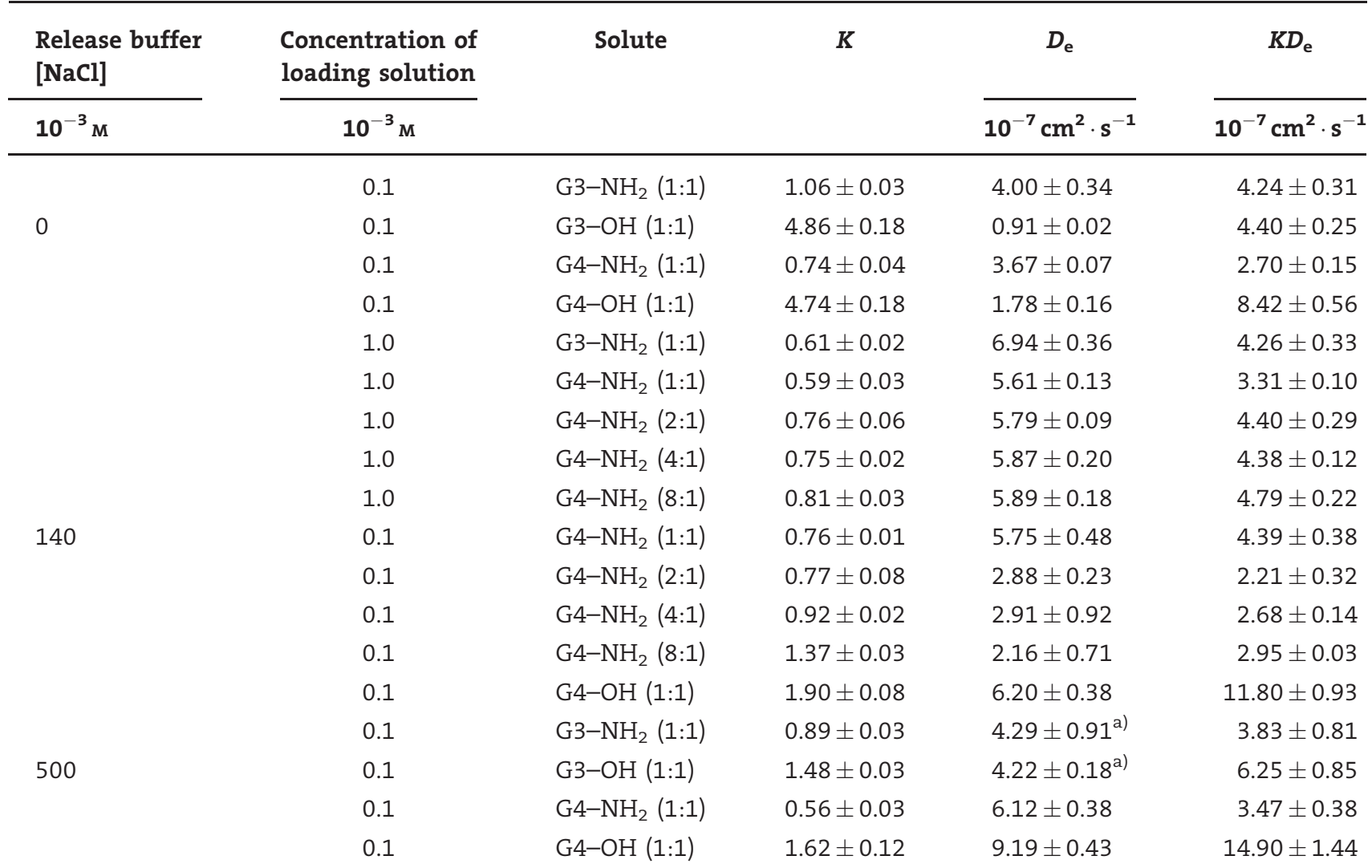

a) Initial $60 \%$ of release data was used in calculating the effective diffusivity.

molecular weight and final extent of FITC labeling of G3-OH and $\mathrm{G} 4-\mathrm{OH}$ was comparable to that found for $\mathrm{G} 3-\mathrm{NH}_{2}$ and $\mathrm{G} 4-\mathrm{NH}_{2}$ dendrimers enabling direct examination of the influence of surface functionality without an appreciable change in molecular size or $K_{\mathrm{ob}}$.

Calculated partition coefficients and apparent diffusivities for $\mathrm{G} 3-\mathrm{NH}_{2}$ (1:1) and G3-OH (1:1) in the presence of high and low salt PBS solutions may be found in Table 5. In the absence of $\mathrm{NaCl}$, the impact of dendrimer net charge on the overall release behavior of $\mathrm{G} 3-\mathrm{NH}_{2}$ (1:1) and $\mathrm{G} 3-\mathrm{OH}$ (1:1) dendrimers is depicted in Figure $3 \mathrm{a}$. In the absence of $\mathrm{NaCl}$, there appears to be a dramatic difference in the partitioning and diffusion behavior of $\mathrm{G} 3-\mathrm{NH}_{2}$ (1:1) and G3-OH (1:1). More than a fourfold increase in partitioning was observed for $\mathrm{G} 3-\mathrm{OH}$ compared to $\mathrm{G} 3-\mathrm{NH}_{2}(1: 1)$ and is accompanied by nearly a fourfold reduction in the effective diffusivity in the SELP-47K network. At high ionic strength conditions $(0.500 \mathrm{M} \mathrm{NaCl})$, partitioning behavior is reduced for both $\mathrm{G} 3-\mathrm{OH}(1: 1)$ and $\mathrm{G} 3-\mathrm{NH}_{2}$ (1:1); however, the effect of increased ionic strength seems to be more prominent for G3-OH (1:1) which resulted in nearly a fourfold decrease in partitioning ability (4.86 compared to 1.48). The calculated effective diffusivities for $\mathrm{G} 3-\mathrm{NH}_{2}$ (1:1) at both low and high ionic strength conditions were very similar indicating that the addition of salt does not appreciably alter the diffusion behavior of $\mathrm{G} 3-\mathrm{NH}_{2}$ (1:1). In comparison, the calculated effective diffusivity for $\mathrm{G} 3-\mathrm{OH}$ (1:1) was nearly fourfold higher as a result of an increase in ionic strength of the release medium. The difference demonstrates the presence of electrostatic interactions with positively charged groups on the polymer chains in the SELP hydrogel similar to those observed with the microsolute fluorescein. The results appear to demonstrate that a change in surface functionality of the dendrimer influences not only its interaction with the hydrogel but its apparent diffusivity in the crosslinked network despite its similar size and hydrophobicity.

The impact of dendrimer net charge on the overall release behavior of $\mathrm{G} 4-\mathrm{NH}_{2}(1: 1)$ and $\mathrm{G} 4-\mathrm{OH}$ (1:1) dendrimers in the absence of $\mathrm{NaCl}$ is depicted in Figure $3 \mathrm{~b}$. The experimental partitioning and diffusion results for G4$\mathrm{NH}_{2}$ (1:1) and G4-OH (1:1) as a function of ionic strength are also presented in Table 5 . An increase in the release medium ionic strength corresponded to an increase in solute effective diffusivities for both $\mathrm{G} 4-\mathrm{NH}_{2}(1: 1)$ and $\mathrm{G} 4-\mathrm{OH}$ (1:1). Solute partition coefficients for G4-OH (1:1) declined 

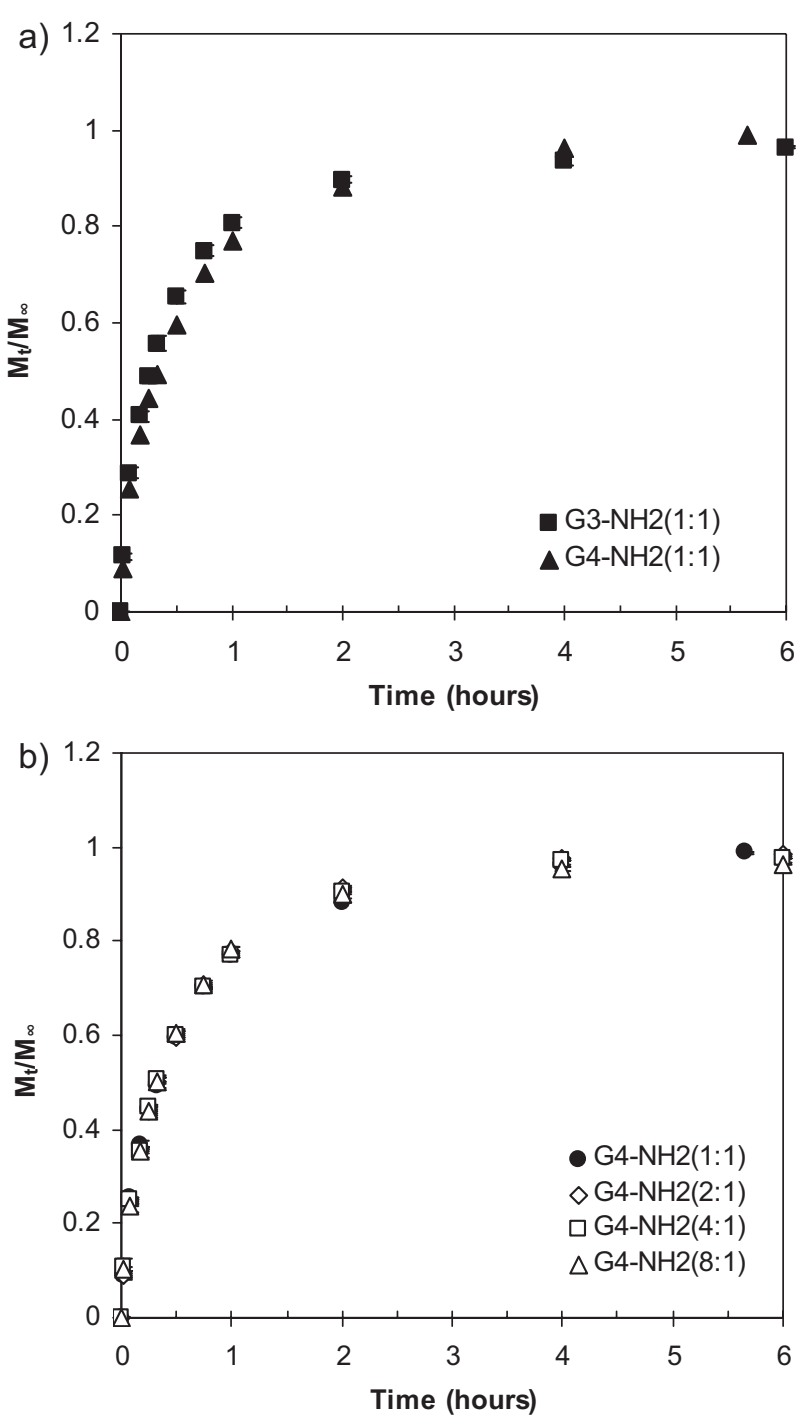

Figure 2. Release kinetics of FITC-labeled $\mathrm{G}_{3}$ and $\mathrm{G}_{4}$ amineterminated dendrimers from SELP-47K hydrogels in PBS ( $\mathrm{pH}=7.4, \mu=0.15$ ). (a) Influence of dendrimer size. (b) Influence of extent of labeling on $\mathrm{G}_{4}$ amine-terminated dendrimer release. All hydrogels equilibrium loaded from $10^{-4} \mathrm{M}$ loading solutions. Symbols represent mean value \pm 1 standard deviation $(n=3)$.

with an increase in ionic strength of the loading solution, while $\mathrm{G} 4-\mathrm{NH}_{2}$ (1:1) partition coefficients demonstrated only a modest decline at $0.500 \mathrm{M} \mathrm{NaCl}$. This finding is most likely due to the fact that FITC is the only substituent that contributes to the net charge of the hydroxyl dendrimers, while FITC substituents on the amine-terminated dendrimers are surrounded by positively charged amine groups. Overall trends associated with the partitioning and diffusion results for $\mathrm{G} 4-\mathrm{NH}_{2}$ (1:1) and $\mathrm{G} 4-\mathrm{OH}$ (1:1) appear to be consistent with results observed for $\mathrm{G} 3-\mathrm{NH}_{2}(1: 1)$ and G3-OH (1:1) dendrimers. However, a closer look at the data for the FITC labeled G4 dendrimers indicates that irrespective of the ionic strength of the release buffer solution,
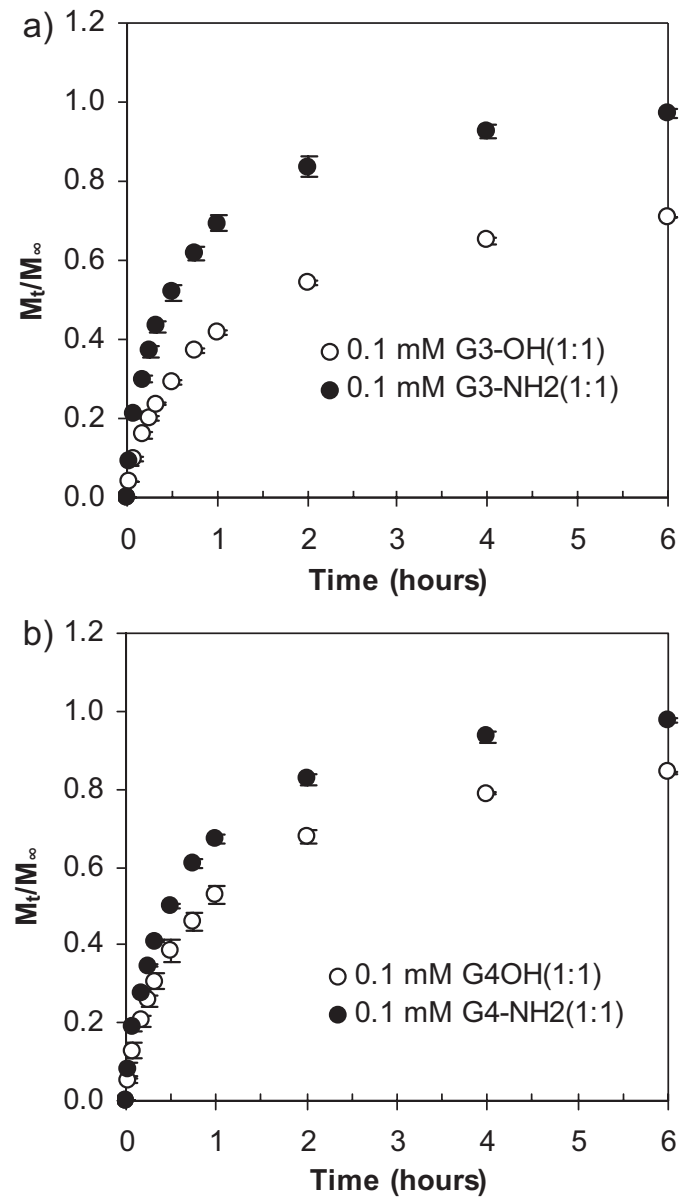

Figure 3. Influence of FITC-labeled $\mathrm{G}_{3}$ and $\mathrm{G}_{4}$ dendrimer surface charge on apparent release kinetics from SELP-47K hydrogels. (a) Comparison of amine and hydroxyl-terminated $\mathrm{G}_{3}$ dendrimers release kinetics in phosphate buffer $(\mathrm{pH}=7.4,[\mathrm{NaCl}]=0)$. (b) Comparison of amine and hydroxyl-terminated $\mathrm{G}_{4}$ dendrimers release kinetics in phosphate buffer $(\mathrm{pH}=7.4,[\mathrm{NaCl}]=0)$. All hydrogels were equilibrium loaded from $10^{-4} \mathrm{M}$ loading solutions. Symbols represent mean value \pm 1 standard deviation $(n=3)$.

effective diffusivities for $\mathrm{G} 4-\mathrm{NH}_{2}(1: 1)$ and $\mathrm{G} 4-\mathrm{OH}(1: 1)$ were higher than those observed for $\mathrm{G} 3-\mathrm{NH}_{2}$ (1:1) or G3-OH (1:1). Using a low-ionic-strength release buffer $([\mathrm{NaCl}]=0)$, estimated effective diffusivities for $\mathrm{G} 3-\mathrm{NH}_{2}$ (1:1) and G4$\mathrm{NH}_{2}$ (1:1) were comparable, yet the effective diffusivity for G4-OH (1:1) was nearly double that observed for G3-OH $(1: 1)\left(1.78 \times 10^{-7}\right.$ compared to $\left.0.91 \times 10^{-7}\right)$. In the absence of $\mathrm{NaCl}$, the lower diffusivity observed for $\mathrm{G} 3-\mathrm{OH}(1: 1)$ compared to $\mathrm{G} 4-\mathrm{OH}(1: 1)$ suggests that $\mathrm{G} 3-\mathrm{OH}(1: 1)$ could have a higher binding affinity with the Polymer $47 \mathrm{~K}$ network, yet at equimolar loading concentrations both G3$\mathrm{OH}(1: 1)$ and G4-OH (1:1) displayed similar partitioning behavior (4.86 and 4.74).

In an attempt to clarify the differences between G3-OH (1:1) and G4-OH (1:1) interaction and diffusion in SELP-47K hydrogels, we examined the influence of $\mathrm{G} 3-\mathrm{OH}(1: 1)$ and 


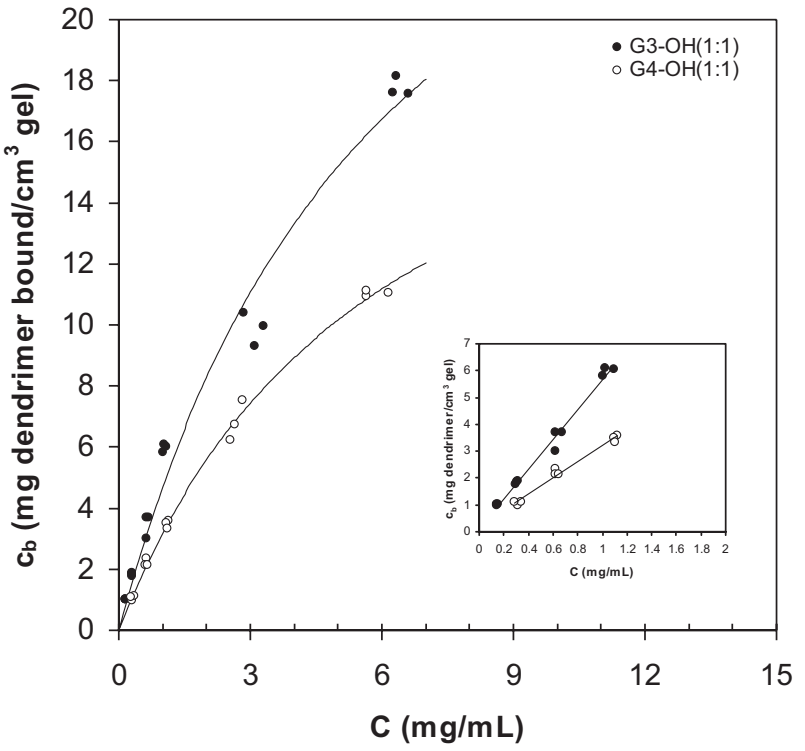

Figure 4. Equilibrium binding behavior of $\mathrm{G}_{3}-\mathrm{OH}(1: 1)$ and $\mathrm{G}_{4}-$ $\mathrm{OH}(1: 1)$ with SELP-47K hydrogels in PBS $(\mathrm{pH}=7.4,[\mathrm{NaCl}]=0)$. Langmuir-type plot along with model fit $(-)$. Inset: dilute solution binding behavior along with model fits for linear binding isotherm (-).

G4-OH (1:1) dendrimer concentration on binding to Polymer 47K hydrogels over a broad concentration range at low ionic strength. The results of the equilibrium binding study are shown in Figure 4 along with model fits corresponding to a Langmuir isotherm and linear binding isotherm (inset). Though the Langmuir model presented in Equation (5) is strictly derived to describe monolayer surface binding for adsorption of solutes dissolved in liquids (or gases) onto solids, it has been successfully used in the literature as an empirical model of ion-exchange binding of macromolecules to hydrogels. ${ }^{[16,17]}$ In Equation (5) and (6), $c_{\mathrm{bm}}$ represents the estimated binding capacity for the solute and $b$ refers to the binding strength of the solute, and $C$ is the concentration of solute in the solution that is in equilibrium with the hydrogel

$$
\begin{aligned}
& c_{\mathrm{b}}=\frac{c_{\mathrm{bm}} b c}{1+b c} \quad \text { Langmuir binding model } \\
& c_{\mathrm{b}}=c_{\mathrm{bm}} b C \text { linear binding model }(\mathrm{bc}<<1)
\end{aligned}
$$

Table 6 presents the estimated model parameters for the Langmuir binding model as well as a linear binding model (dilute solution limit). The estimated parameters for the Langmuir model indicate that $\mathrm{G} 3-\mathrm{OH}(1: 1)$ has a greater binding capacity compared to G4-OH (1:1) although the estimated binding strength of each FITC labeled dendrimer is essentially the same. The data suggest that differences in the diffusion behavior of $\mathrm{G} 3-\mathrm{OH}(1: 1)$ and $\mathrm{G} 4-\mathrm{OH}(1: 1)$ may
Table 6. Equilibrium binding parameters for FITC-labeled $\mathrm{G}_{3}-\mathrm{OH}$

\begin{tabular}{|c|c|c|c|}
\hline \multirow[t]{3}{*}{ Solute } & \multicolumn{2}{|c|}{ Langmuir isotherm } & \multirow{3}{*}{$\frac{\begin{array}{c}\text { Linear } \\
\text { isotherm }\end{array}}{c_{\mathrm{bm}} b}$} \\
\hline & $c_{\mathrm{bm}}$ & $b$ & \\
\hline & $\mathbf{m g} \cdot \mathbf{m L}^{-1}$ & $\mathbf{m L} \cdot \mathbf{m g}^{-1}$ & \\
\hline G3-OH (1:1) & 34.28 & 0.16 & 5.52 \\
\hline G4-OH (1:1) & 22.47 & 0.17 & 2.99 \\
\hline
\end{tabular}
and $\mathrm{G}_{4}-\mathrm{OH}$ PAMAM dendrimers.

All studies performed using $10^{-2} \mathrm{M}$ phosphate buffer, $\mathrm{pH}=7.4$, $[\mathrm{NaCl}]=0$.

be related to differences in the amount of sites available for binding as well as their individual binding behavior.

\section{Comparison of $\mathrm{G}_{3} \mathrm{OH}$ (1:1) and $\mathrm{G}_{4} \mathrm{OH}$ (1:1) Release Data With a Pore Diffusion Transport Model with Instantaneous Desorption}

A simple method of modeling solute transport in hydrogels is to envision the hydrogel as a porous medium where the diffusivity of a given solute in the hydrogel is influenced by both the network porosity $(\varepsilon)$ and the tortuosity $(\tau)$ of the system of pores in the hydrogel. An effective diffusivity for transport in porous media may be defined by Equation (7), where $D_{0}$ represents the aqueous solute diffusivity at a given set of experimental conditions

$$
D_{e, p}=\frac{D_{0} \varepsilon}{\tau}
$$

If transport of the hydrophilic PAMAM dendrimers is believed to occur only in the water-filled voids of the hydrogel, intra-gel transport may be modeled by Equation (8) under the following assumptions: (1) solute transport occurs only in the bulk water regions of the hydrogel (pore diffusion), (2) solutes that interact with the polymer chains of the hydrogel are immobilized in the network (i.e., do not retain diffusional mobility in the bound state), (3) there is an initial, homogeneous distribution of solute in the hydrogel following equilibrium loading where any solute interacting with the hydrogel is in equilibrium with free solute in the hydrogel network, (4) upon release into an infinite sink, bound solutes are instantaneously desorbed followed by diffusion in the water-filled pores. In the past, this mechanism has been successfully used to model intra-particle solute transport in water-filled, crosslinked polymer supports used as chromatography media. ${ }^{[17,18]}$ This mechanism also appears to be physically consistent with scanning electron microscopy (SEM) 
images (data not shown) as well as from visual observations made during the release studies for G3-OH (1:1) and G4-OH (1:1) at low ionic strength:

$$
\varepsilon \frac{\partial c}{\partial t}+\frac{\partial c_{b}}{\partial t}=D_{e, p} \nabla^{2} c
$$

where $c_{b}$ represents the concentration of solute bound to the hydrogel network, $c$ is the solute concentration in the water-filled pores, $D_{\mathrm{e}, \mathrm{p}}$ is the average solute diffusion coefficient in the hydrogel pores, and $\varepsilon$ is the porosity of the hydrogel (estimated as the volume fraction of bulk water in the hydrogel). If we assume that local equilibrium exists at the pore surface and the surrounding fluid, then $c_{b}$ may be expressed in terms of $C$ by an equilibrium uptake isotherm with fitted parameters at a given set of experimental conditions. In the simple case of a linear equilibrium uptake isotherm, $c_{b}$ is related to $C$ by an equilibrium parameter $c_{\mathrm{bm}} b$ that is independent of concentration for a given solute. It is assumed that the concentration of free solute in the hydrogel pores $(c)$ is essentially the same as that found in the bulk liquid at the surface of the hydrogel $(C)$ post-equilibration. The rate of change of bound polymer $\left(c_{b}\right)$ with respect to time may then be related to the equilibrium uptake parameters via Equation (9)

$$
\frac{\partial c_{\mathrm{b}}}{\partial t}=\frac{\mathrm{d} c_{\mathrm{b}}}{\mathrm{d} c}\left(\frac{\partial c}{\partial t}\right)=c_{\mathrm{bm}} b \frac{\partial c}{\partial t}
$$

Substituting Equation (9) on the left-hand side of Equation (8) and re-arranging yields Equation (10),

$$
\frac{\partial c}{\partial t}=\left(\frac{D_{\mathrm{e}, \mathrm{p}}}{\varepsilon+c_{\mathrm{bm}} b}\right) \nabla^{2} c
$$

If we express the Laplacian of the free solute concentration $\left(\Delta^{2} c\right)$ in terms of two-dimensional diffusion in a tablet geometry ( $r$ and $z$ direction only), we arrive at the differential Equation that corresponds to the solution given in Equation (2) where the average intra-gel diffusivity, $D_{\mathrm{e}}$ corresponds to the term found in Equation (11),

$$
D_{\mathrm{e}}=\left(\frac{D_{\mathrm{e}, \mathrm{p}}}{\varepsilon+c_{\mathrm{bm}} b}\right)
$$

Figure $5 \mathrm{a}$ and $\mathrm{b}$ compare the pore diffusion model utilizing the equilibrium binding parameters with the experimental initial release profiles for $\mathrm{G} 3-\mathrm{OH}(1: 1)$ and G4-OH (1:1) from SELP-47K hydrogels under low ionic strength conditions. Aqueous diffusivities used in the pore diffusion model for $\mathrm{G} 3-\mathrm{OH}(1: 1)$ and $\mathrm{G} 4-\mathrm{OH}$ (1:1) were estimated based on the theoretical molecular weight of the unlabeled polymer and may be considered as upper-
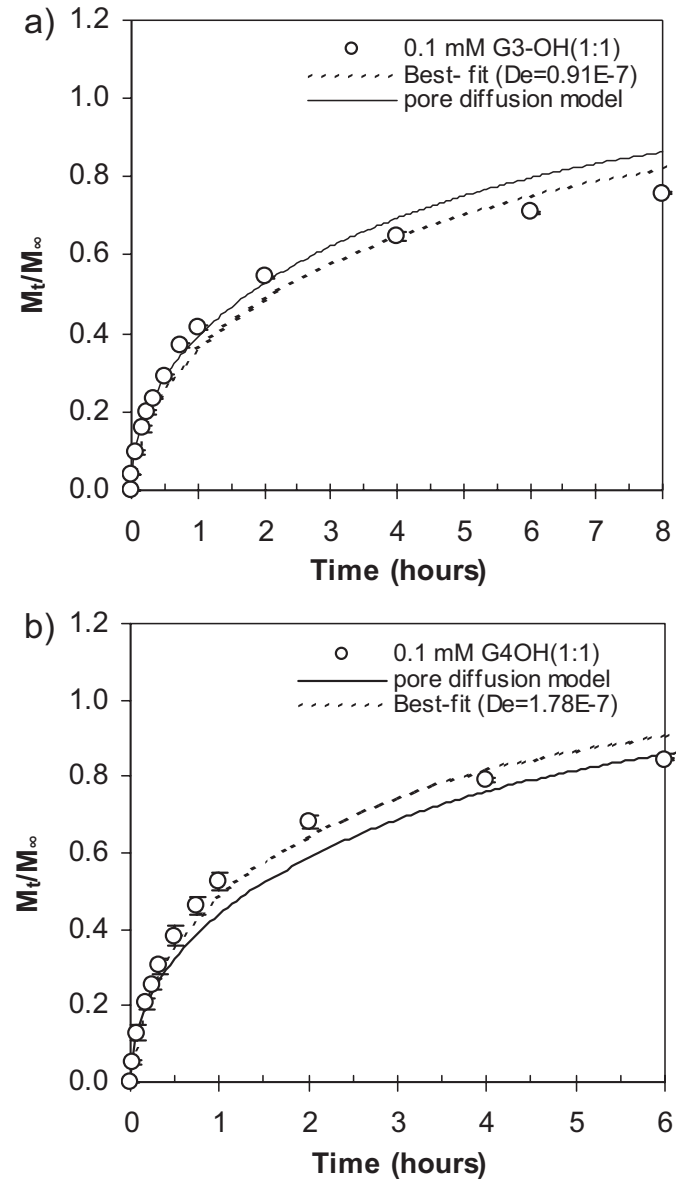

Figure 5. Comparison of pore diffusion model with: (a) $\mathrm{G}_{3}-\mathrm{OH}(1: 1)$ and (b) $\mathrm{G}_{4}-\mathrm{OH}(1: 1)$ initial release kinetics in phosphate buffer $(\mathrm{pH}=7.4,[\mathrm{NaCl}]=0)$. Symbols represent mean value \pm 1 standard deviation $(n=3)$.

bound estimates of the true aqueous diffusivity of the FITC labeled-polymers (see Table 4). A tortuosity value of 2.4 ( $\tau=2.4$ ) was used for the pore diffusion model calculations and was estimated from previous solute diffusion data in networks formed after a $24 \mathrm{~h}$ cure time for $12 \mathrm{wt}$.-\% Polymer $47 \mathrm{~K}$ solutions. ${ }^{[9]}$ As shown in Figure $5 \mathrm{a}$ and $\mathrm{b}$, despite its simplicity the pore diffusion model (with no adjustable parameters) provides a reasonable prediction of the initial experimental release data by accounting for the electrostatic interaction observed at low ionic strength for $\mathrm{G} 3-\mathrm{OH}(1: 1)$ and $\mathrm{G} 4-\mathrm{OH}(1: 1)$.

Recombinant SELP hydrogels have shown promise in controlled delivery of bioactive agents. ${ }^{[19]}$ Recent work for example points to the influence of exquisite control over polymer structure leading to control over localized adenoviral gene delivery and transfection in head and neck solid tumors. ${ }^{\left[{ }^{20]}\right.}$ In addition these polymers have shown promise in tissue engineering applications. ${ }^{[21]}$ In both cases concomitant delivery of small molecular weight drugs such as chemotherapeutics, or large molecular weight compounds 
such as growth factors are essential to complement the activity of the anticancer genes (in the case of gene therapy), or promote growth and spreading of cells (in case of tissue engineering). The studies presented herein provide important information about the influence of size, charge and hydrophobicity of compounds on diffusion in a given SELP hydrogel. By tailor making polymer structure it is possible to modify the release behavior of solutes and hence design recombinant SELPs that control release of bioactive agents with specific physicochemical properties for desired applications.

\section{Conclusion}

The influence of solute charge and hydrophobicity on solute partitioning and diffusion in physically crosslinked networks of a genetically engineered silk-elastin-like polymer was investigated. The partitioning and diffusion of a series of fluorescent dyes of similar molecular size demonstrated that variations in solute hydrophobicity influence partitioning and release behavior from the SELP hydrogel under study. The release kinetics of fluorescein appears to represent a Fickian dominant release mechanism. In contrast, pseudo-Fickian release behavior was observed for rhodamine B and rhodamine $6 \mathrm{G}$, which may suggest competing dye transport rates in both the rigid polymer and bulk water portions of the SELP hydrogel. The release behavior of the small molecular weight anion fluorescein was influenced by the ionic strength of the release medium further substantiating the evidence of an electrostatic interaction previously observed with plasmid DNA released from direct-loaded SELP-47K hydrogels. The strong partitioning behavior of rhodamine $B$ and rhodamine $6 \mathrm{G}$ compared to fluorescein in the presence of $0.140 \mathrm{M} \mathrm{NaCl}$ indicates that functional group modifications of small molecules may have a pronounced effect on solute permeability and transport mechanisms in this SELP hydrogel. The transport and partitioning properties of fluorescent labeled G3 and G4 PAMAM dendrimers demonstrated similar results. The partitioning and diffusion of amine terminated PAMAM dendrimers $\left(\mathrm{G} 3-\mathrm{NH}_{2}\right.$ and $\mathrm{G} 4-$ $\mathrm{NH}_{2}$ ) appeared to be a function of both the extent of FITC labeling of functional groups on the surface of the dendrimers as well as the loading concentration of highly labeled dendrimer solutions. Hydrogel affinity was more related to the extent of functional groups substituted with FITC rather than due to changes in FITC labeled dendrimer hydrophobicity. Results suggest that small changes in the net surface charge of the hydrophilic dendrimers primarily influenced solute partitioning although all of the probes theoretically retained an overall positive charge at the experimental conditions. Comparison of the transport and partitioning behavior of the amine-terminated dendrimers with FITClabeled hydroxyl terminated dendrimers (G3-OH and G4-
$\mathrm{OH}$ ) suggest that the attachment of hydrophobic solutes such as FITC to the surface functional groups impact solute uptake and transport behavior in SELP-47K hydrogels. Observed differences in partitioning and diffusion behavior between the amine terminated and hydroxyl terminated dendrimers was primarily due to a larger net negative surface charge for the hydroxylated dendrimers as a result of only the FITC labeled surface groups contributing to the net charge of the macromolecule. SELP-47K hydrogels appeared to prefer interactions with $\mathrm{G} 3-\mathrm{OH}(1: 1)$ over the concentration range in which the studies were performed. This strong preference was confirmed by equilibrium uptake studies and seemed to be associated with differences in binding capacity and binding behavior when compared to $\mathrm{G} 4-\mathrm{OH}$ (1:1). Using the equilibrium uptake parameters calculated from a linear binding isotherm for the hydroxylated dendrimers, a pore diffusion model combined with instantaneous desorption of bound solute was used to successfully predict the initial transport rates of hydrophilic FITC labeled G3-OH and G4-OH dendrimers in SELP$47 \mathrm{~K}$ hydrogels under low ionic strength conditions. Results of this study can be useful for the design and development of genetically engineered SELPs for controlled delivery of therapeutic macromolecules such as polymer-drug conjugates, proteins, and nucleic acids.

Acknowledgements: This work was partially funded by an American Foundation for Pharmaceutical Education (AFPE) Fellowship for AAD, and grants from the Department of Defense Breast Cancer Research Program (DAMD 17-03-0237) and the National Institutes of Health (R01CA107621). The authors thank Ms. Steffi Sunny for reviewing and formatting the manuscript. Support from Jordan Frandsen and Clark Newell in graphics is appreciated.

Received: February 10, 2010; Revised: April 10, 2010; Published online: July 2, 2010; DOI: 10.1002/mabi.201000061

Keywords: dendrimers; diffusion; drug delivery; genetically engineered polymers; hydrogels

[1] J. Cappello, J. W. Crissman, M. Crissman, F. A. Ferrari, G. Textor, O. Wallis, J. R. Whitledge, X. Zhou, D. Burman, L. Aukerman, E. R. Stedronsky, J. Controlled Release 1998, 53, 105.

[2] R. A. McMillan, V. P. Conticello, Macromolecules 2000, 33, 4809.

[3] W. A. Petka, J. L. Harden, K. P. McGrath, D. Wirtz, D. A. Tirrell, Science 1998, 281, 389.

[4] J. Lee, C. W. Macosko, D. W. Urry, Macromolecules 2001, 34, 4114.

[5] D. W. Urry, C. M. Harris, C. X. Luan, C. Luan, "Controlled Drug Delivery-Strategies and Challenges", American Chemical Society, Washington 1997, p. 405. 
[6] M. Haider, Z. Megeed, H. Ghandehari, J. Controlled Release 2004, 95, 1.

[7] Z. Megeed, J. Cappello, H. Ghandehari, Adv. Drug Delivery Rev. 2002, 54, 1075.

[8] A. A. Dinerman, J. Cappello, H. Ghandehari, S. W. Hoag, Biomaterials 2002, 23, 4203.

[9] A. A. Dinerman, J. Cappello, H. Ghandehari, S. W. Hoag, J. Controlled Release 2002, 82, 277.

[10] Z. Megeed, J. Cappello, H. Ghandehari, Pharm. Res. 2002, 19, 954.

[11] D. A. Tomalia, A. M. Naylor, W. A. Goddard, Angew. Chem. Int. Ed. Engl. 1990, 29, 138.

[12] M. El-Sayed, M. F. Kiani, M. D. Naimark, A. H. Hikal, H. Ghandehari, Pharm. Res. 2001, 18, 23.

[13] J. C. Fu, C. Hagemeir, D. L. Moyer, E. W. Ng, J. Biomed. Mater. Res. 1976, 10, 743.
[14] J. Siepmann, A. Ainaoui, J. M. Vergnaud, R. Bodmeier, J. Pharm. Sci. 1998, 87, 827.

[15] P. L. Ritger, N. A. Peppas, J. Controlled Release 1987, 5, 37.

[16] M. P. Singh, J. A. Lumpkin, J. Rosenblatt, J. Controlled Release 1995, 35, 165.

[17] L. E. Weaver, G. Carta, Biotechnol. Prog. 1996, 12, 342.

[18] H. Komiyama, J. M. Smith, AIChE J. 1974, 20, 728.

[19] R. Dandu, H. Ghandehari, Prog. Polym. Sci. 2007, 32, 1008.

[20] J. Gustafson, K. Greish, J. Frandsen, J. Cappello, H. Ghandehari, J. Controlled Release 2009, 140, 256.

[21] M. Haider, J. Cappello, H. Ghandehari, K. W. Leong, Pharm. Res. 2008, 25, 692.

[22] M. Araie, D. Maurice, Exp. Eye Res. 1987, 44, 73.

[23] W. M. Saltzman, M. L. Radomsky, K. J. Whaley, R. A. Cone, Biophys. J. 1994, 66, 508. 